\title{
MECONIUM AND FAECAL PLUGS IN THE NEWBORN*
}

\author{
BY \\ R. B. ZACHARY \\ From the Children's Hospital, Sheffield
}

This paper deals with the clinical aspects of a series of cases, the pathology of which has been described in the preceding paper by Emery. The cases fall into two distinct groups but some relationship between these groups will be discussed later.

The first group consists of cases of rectal obstruction by meconium plugs. In the second group the obstruction was due to hard faeces and was complicated by stercoral ulceration.

\section{Meconium Plugs}

Paediatricians and many general practitioners have been made aware that surgery has much to offer in the treatment of intestinal obstruction of the newborn and many are on the look-out for the cardinal symptom of vomiting green material in the first 12 hours of life. Any child with this symptom should be suspected of having intestinal obstruction. If in addition the infant has not passed meconium in the first 24 hours or so, or has passed some abnormal meconium, there is a very strong presumption of obstruction and the child should be sent to a centre where the paediatric and surgical attention can be expected to be of a high standard.

It is not surprising that a number of cases presenting in this way have turned out to be something different from the usual types of neonatal obstruction, atresia, stenosis, volvulus, meconium ileus and Hirschsprung's disease. They have been obstructed by a terminal plug of meconium. Brief clinical details of four of these cases are as follows.

Case 1. An infant was admitted at the age of $\mathbf{4 0}$ hours with a history of vomiting bile-stained material since birth; no meconium had been passed. On examination the infant was fairly well, not dehydrated and had a somewhat full abdomen. Rectal examination produced a plug of thick meconium which was followed by a large quantity of normal meconium; the child had normal bowel action afterwards and was well.

Case 2. An infant was admitted at the age of 36 hours, vomiting since birth, and the vomitus had become brown.

\footnotetext{
*A paper read at the meeting of the British Association of Paediatric Surgeons in London in July, 1956.
}

No meconium had been passed. The infant was well apart from some abdominal distension and rectal examination produced a similar plug of meconium after which bowel action was normal. Recently a sweat test was done on this child and the result was within the normal range.

Case 3. This infant was somewhat older when admitted, 6 days, but had been vomiting from the age of 4 hours and had only passed one meconium stool (and that a small one) at 36 hours. This child was not well, moderately dehydrated, with a full abdomen. Rectal examination produced nothing at all but a rectal wash-out yielded some plugs of meconium, after which bowel action was normal.

Case 4. A 32-hour-old infant was admitted who had repeatedly vomited brown material and had not passed meconium. The abdomen was full, the child moderately ill, and rectal examination did not reveal any faecal matter. Preparations were made for operation with a diagnosis of intestinal obstruction, but the child suddenly died. Necropsy showed that death was due to inhalation, resulting from intestinal obstruction by a meconium plug in the rectum.

More than $90 \%$ of newborn infants pass meconium within the first 24 hours of life and probably another 7 or $8 \%$ in the next 12 hours, and the only difference between the remaining 2 or $3 \%$ and the cases described above is that the latter were vomiting bile-stained material and were therefore in danger of death by inhalation. The plug found in these cases was similar to that normally passed when the rectum is first emptied and to that found in the stillborn, but with a lower water content. It seems likely that the greater firmness of this plug is due to its remaining longer in the rectum rather than to any intrinsic abnormality of the meconium.

\section{Faecal Plugs with Stercoral Ulceration}

In the second group of cases the infants were admitted to hospital between 4 days and 5 weeks of age and were found to have a perforation of the colon overlying a hard mass of faeces. The following are brief summaries of six cases. 
Case 5. This infant was admitted at the age of 4 days with a history of vomiting from the second day; meconium had been passed at 30 hours and also on the third day. The child was very ill, vomiting brown material with a distended abdomen. Radiographs showed free gas under the diaphragm. At operation a perforation of the sigmoid colon was found and the loop exteriorized. The child died shortly afterwards and at necropsy the sigmoid was deep red with some necrotic zones overlying faecal matter. The pancreas was normal and histology of the colon showed ganglion cells throughout.

Case 6. An infant of 5 days of age was admitted who had vomited about 15 minutes after every feed and had only passed two small meconium stools. After admission the child passed a few small loose brown stools for two days and then quite quickly became seriously ill, vomiting faecal contents and died suddenly. Necropsy revealed perforation of the ascending colon with a terminal respiratory infection. The histology of the pancreas and the colon were normal.

Case 7. This child came into hospital at the age of 9 days with increasing constipation and abdominal distension. Radiographs showed free gas in the abdomen. At operation no perforation was found and a transverse colostomy was made in the belief that Hirschsprung's disease was the cause. He died at the age of $\mathbf{1 5}$ days from peritonitis due to a perforation of the ascending colon and a similar perforation of the splenic flexure, distal to the colostomy. Histology showed ganglion cells in the colon and a normal pancreas.

Case 8. This child was sent into hospital by a paediatrician at the age of 7 days because she had vomited repeatedly some yellow fluid after feeds, and, although she passed four normal-looking meconium stools during the first 24 hours, there was no further spontaneous bowel movement. The infant was moderately ill, with a distended abdomen but shortly after rectal examination meconium was passed (quite obvious greenish black material), followed by a lot of gas and later softish faeces. She was sent home after four days but was admitted three days later moribund. She was vomiting yellowish-brown fluid and had not passed a stool since discharge. She was extremely dehydrated, with a distended abdomen, and rectal examination showed a small amount of hard faeces. She died within a few hours and necropsy revealed peritonitis due to perforation of the sigmoid colon overlying a mass of hard faeces. The pancreas was normal and ganglion cells were present in the distal colon.

Case 9. This infant had originally come into hospital at the age of 1 day because of abdominal distension and failure to pass meconium. Rectal examination produced some normal meconium and the child went home quite well. He was readmitted at the age of 4 weeks because he was underweight and the feeding régime was increased. Two days later he suddenly became extremely ill with vomiting and distension of the abdomen. At operation a perforation of the sigmoid colon was found and the loop was exteriorized. The child died and was found to have peritonitis and a gross congenital heart lesion. The pancreas was normal and the intramural plexus of the colon was quite normal.

Case 10. This was an infant of 5 weeks who had vomited fairly frequently since birth and had hard stools for some days before admission. The child had a renal tract infection which was treated with antibiotics and an enema produced some very hard faecal material. After two weeks in hospital the child became very ill, vomiting, with abdominal distension and not passing stools. The baby was thought to have Hirschsprung's disease, an impression which was enhanced at operation when a distended colon was found. A transverse colostomy was performed but the child died suddenly a few days later and was found to have a perforation of the sigmoid colon over some very hard faecal matter. Histology showed a normal pancreas and a careful study of the colon failed to confirm the impression of Hirschsprung's disease.

\section{Discussion}

Although this second group of cases is in some ways so different from the first, there are some findings in common in a few of the infants. One child in the first group (Case 3) passed meconium at 36 hours but nothing more until a rectal wash-out was given at 6 days. Clearly something more than a terminal ano-rectal plug was involved. Moreover, in the second group were several infants who passed little or no meconium in the first few days, and there must have been delay or difficulty in expelling meconium from the colon.

In the first group the possibility of a mild form of meconium ileus was considered but tryptic activity was present at a high dilution in the stools, and in the one case where a sweat test was done there was no evidence of an increased loss of salt.

Hirschsprung's disease was eliminated by the smooth course once the rectal plug was removed.

The most likely diagnosis in the second group of these cases seemed to be Hirschsprung's disease and on that account particular attention was paid to the intramural plexus at necropsy but no abnormality was found. The most striking features have been the hardness of the faecal contents and the fact that the perforation was usually overlying a hard faecal mass. In two cases deflation of the bowel proximal to this point had not prevented ulceration.

Perforation could have been due to a congenital defect in one segment of the bowel wall but no such defect was found. It seems more likely that there was a pressure necrosis of a normal bowel by a hard faecal mass and that the firmness of the faecal matter was due to excessive absorption of water. Excessive water take-up might be a peculiarity of the bowel wall but it is much more likely to be due to 
delay in the passage of the contents of the colon on towards the rectum and then through the anus and it might be that some incoordination of large bowel action in these babies is a manifestation of immaturity of function.

\section{Summary}

Ten cases are described in which there was obstruction of the large bowel in the first few weeks of life.

Four of these cases come clearly under the heading of meconium plugs which can be relieved by rectal examination or rectal wash-out in a child who presents in the neonatal period as intestinal obstruction. In six cases there was incomplete obstruction of the colon by hard faecal matter between the ages of 4 days and 5 weeks and perforation of the bowel occurred in all cases.

Thanks are due to Dr. T. Colver, Dr. R. Gordon and Professor R. S. Illingworth for permission to include cases admitted under their care. 